\title{
MORTALIDAD POR ENFERMEDAD RENAL CRÓNICA EN EL PERÚ: TENDENCIAS NACIONALES 2003-2015
}

\author{
RESUMEN
}

\author{
Rodrigo M. Carrillo-Larco ${ }^{1,2, a}$, Antonio Bernabé-Ortiz $2,3, \mathrm{~b}$
}

\begin{abstract}
Objetivos. Describir la tendencia de mortalidad por enfermedad renal crónica (ERC) en el Perú en el periodo 2003-2015. Materiales y Métodos. Análisis de datos secundarios y de diseño ecológico. Se analizaron registros nacionales de mortalidad basados en certificados de defunción. ERC fue definida como código CIE 10: N18 en la causa básica. Se describen frecuencias absolutas y relativas de muerte por ERC. Para estimar la proporción de muertes por ERC en cada región del Perú, se utilizó un modelo lineal mixto generalizado. La variable desenlace fue la proporción de muertes, las variables independientes fueron cada región del país y el año. Resultados. El análisis incluyó 1086778 defunciones, de los cuales 25091 (2,0\% ajustado por edad y sexo) fueron por ERC. Durante el periodo de estudio la edad promedio, al momento del fallecimiento, aumentó en 2,6 años ( $p<0,001)$; además, la proporción de muertes por ERC ajustada por edad y sexo fue siempre mayor en mujeres. En el periodo de observación la región con mayor mortalidad por ERC fue Puno (4,1\%), y con menor mortalidad fue Amazonas (1,1 $\%)$. Aquellas regiones que mostraron un aumento significativamente mayor que las demás fueron Tacna, La Libertad, Tumbes, Apurímac, Cusco, Ica, Moquegua, Ayacucho, Huancavelica y Puno. Conclusiones. En el periodo 2003-2015, la mortalidad por ERC en el Perú ha aumentado; esta tendencia se observa en varias regiones del país. Es necesario implementar medidas de prevención, temprana identificación, y acceso al tratamiento, para mejorar esta tendencia.
\end{abstract}

Palabras clave: Insuficiencia renal crónica; Mortalidad; Perú (fuente: DeCS BIREME)

\section{MORTALITY FROM CHRONIC KIDNEY DISEASE IN PERU: NATIONAL TRENDS 2003-2015}

\begin{abstract}
Objectives. To describe the mortality trend due to chronic kidney disease (CKD) in Peru in the period 2003-2015. Materials and Methods. Analysis of secondary data and ecological design. National mortality registries based on death certificates were analyzed. ERC was defined as CIE code 10: N18 in the basic cause. Absolute and relative frequencies of death by ERC are described. To estimate the proportion of deaths by CKD in each region of Peru, a generalized mixed linear model was used. The outcome variable was the proportion of deaths, the independent variables were each region of the country and the year. Results. The analysis included 1,086,778 deaths, of which 25,091 (2.0\% age- and sex-adjusted) were by CKD. During the study period, the average age at death increased by 2.6 years $(p<0.001)$; in addition, the proportion of age- and sex-adjusted CKD deaths was always higher in women. In the observation period, the region with the highest mortality from CKD was Puno (4.1\%), and with the lowest mortality was Amazonas (1.1\%). Those regions that showed a significantly greater increase than the others were Tacna, La Libertad, Tumbes, Apurímac, Cusco, Ica, Moquegua, Ayacucho, Huancavelica and Puno. Conclusions. In the 2003-2015 period, mortality from CKD in Peru has increased; this trend is observed in several regions of the country. Preventive measures, early identification, and access to treatment must be implemented to control this trend.
\end{abstract}

Keywords: Chronic kidney disease; Mortality; Perú (source: DeCS BIREME)

\section{INTRODUCCIÓN}

La enfermedad renal crónica (ERC) está entre las veinte primeras causas de años de vida perdidos a nivel global ${ }^{(1)}, y$ su efecto en los años de vida con discapacidad tampoco es despreciable ${ }^{(2)}$. A pesar de ser una enfermedad importante por la alta mortalidad, discapacidad y costos asociados, la investigación local sobre ella es escasa ${ }^{(3)}$. La evidencia más reciente indica que la prevalencia de $\mathrm{ERC}$ a nivel poblacional en el Perú es $16 \%{ }^{(4)}$. Sin embargo, este estimado no es a nivel nacional, dejando pendiente dilucidar si este resultado varía en diferentes partes del país. Dado que los principales

\footnotetext{
Department of Epidemiology and Biostatistics, School of Public Health, Imperial College London. London, UK.

CRONICAS Centro de Excelencia en Enfermedades Crónicas, Universidad Peruana Cayetano Heredia. Lima, Perú.

Facultad de Ciencias de la Salud, Universidad Peruana de Ciencias Aplicadas. Lima, Perú.

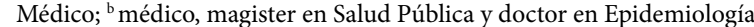

Recibido: 22/04/2018 Aprobado: 05/09/2018 En línea: 25/09/2018
}

Citar como: Carrillo-Larco RM, Bernabé-Ortiz A. Mortalidad por enfermedad renal crónica en el Perú: tendencias nacionales 2003-2015. Rev Peru Med Exp Salud Publica. 2018;35(3):409-15. doi:. 10.17843/rpmesp.2018.353.3633. 
factores de riesgo para la ERC (p. ej. diabetes mellitus tipo 2 e hipertensión arterial) se distribuyen de distinta manera en el Perú (5-9), se podría deducir que la prevalencia de ERC no sería similar en todas las regiones, y tampoco lo sería la mortalidad asociada. Desafortunadamente, la evidencia sobre mortalidad se ha enfocado principalmente en pacientes en terapias de reemplazo renal (3). No obstante, un reporte del Ministerio de Salud (MINSA) señala que, en el periodo 2000-2012, las regiones con mayor mortalidad por ERC fueron de la sierra del Perú ${ }^{(10)}$. Así pues, para complementar la evidencia disponible a nivel hospitalario y dar más información sobre la cual plantear estudios o intervenciones para entender o disminuir la mortalidad por ERC, el objetivo del presente estudio fue describir la mortalidad por ERC, y el cambio en el tiempo de esta, entre los años 2003 y 2015 en cada región del Perú.

\section{MATERIALES Y MÉTODOS}

\section{DISEÑO DE ESTUDIO Y FUENTE DE DATOS}

Análisis de datos secundarios y de diseño ecológico. Se analizaron registros nacionales de mortalidad, basados en certificados de defunción, correspondientes al periodo 20032015 proporcionados por el Ministerio de Salud del Perú (MINSA). Los registros contienen todos los fallecimientos ocurridos y registrados en el país, incluyendo aquellos sucedidos en establecimientos de salud y fuera de ellos (p. ej. ambulancia). Sin embargo, a pesar de ser datos nacionales, puede haber subreporte. Estos registros están disponibles y pueden ser solicitados a través del portal de acceso a la información pública del MINSA (https://goo.gl/GhPbtW). En este estudio solo se incluyeron registros con información completa de la ubicación del fallecimiento (p. ej. región) y a sujetos de mayores de 18 años.

\section{VARIABLES}

La variable de interés fue la mortalidad por enfermedad renal crónica (ERC), definida de acuerdo a la causa básica de muerte usando el CIE 10 (código N18). Según disponibilidad de los datos, las otras variables incluidas fueron sexo, edad al momento del fallecimiento, y región habitual del sujeto (25 regiones del Perú: 24 departamentos y una provincia constitucional).

\section{ANÁLISIS ESTADÍSTICOS}

El análisis de datos se hizo según lo registrado, sin haber realizado previamente correcciones por defunciones no certificadas. Los análisis estadísticos se hicieron en STATA 13.0 (StataCorp, College Station, TX, USA) y en el paquete estadístico $R{ }^{(11)}$. Se estimaron frecuencias absolutas $y$ relativas, para toda la población, según sexo y grupo etario, en total y para cada año de observación. Se evaluó tendencias lineales (nptrend en STATA) para evaluar si hubo un aumento

\section{MENSAJES CLAVE}

Motivación para realizar el estudio. En el Perúl la enfermedad renal crónica es una causa importante de muerte. Sin embargo, no se han estudiado las tendencias de mortalidad en el tiempo, según regiones o en población general, en comparación con estudios en pacientes en tratamiento o población hospitalaria.

Principales hallazgos. En el periodo 2003-2015, la mortalidad por enfermedad renal crónica, en números absolutos en relación a otras causas, ha aumentado. Esta tendencia se cumple en varias regiones, como Huancavelica y Puno (regiones de altura).

Implicancias. Los resultados sugieren que existen particularidades en cada región que determinan distintas cifras de mortalidad; éstas deben estudiarse, identificarse y abordarse para mejorar las tendencias reportadas.

en la edad de fallecimiento por ERC. La proporción de muerte por ERC se presenta ajustada por edad y sexo utilizando como referencia la población de la Organización Mundial de la Salud 2000-2025 (https://goo.gl/shkDQq).

Para representar las tendencias de mortalidad por ERC a través de los años se siguió una aproximación ecológica a través de un modelo lineal mixto (xtmixed en STATA). La unidad de análisis fue una región-año (p. ej. Lima-2005). La variable desenlace fue la proporción de muertes por ERC en cada región y en cada año. Se construyeron 25 variables dummy, una por cada región (p. ej. 1=Lima, $0=$ no Lima); estas 25 variables fueron incluidas en el modelo de regresión como variables independientes. Asimismo, el modelo incluyó el año, la proporción de mujeres fallecidas en cada región y en cada año, y la proporción de adultos mayores (65+ años) fallecidos en cada región y en cada año; una aproximación ajustada por sexo y edad. El modelo además incluyó un intercepto aleatorio, definido por la región y se asumió una covarianza no estructurada. El valor estimado para cada variable dummy se utilizó para estimar las tendencias.

\section{CONSIDERACIONES ÉTICAS}

Se trata de un análisis de datos secundarios de información anónima. No hubo riesgo de identificar a los sujetos de estudio.

\section{RESULTADOS}

\section{BASE DE DATOS}

Entre 2003 y 2015 se registraron 1202386 fallecimientos. Luego de excluir observaciones sin información de la región habitual del sujeto $(n=570)$, y sujetos menores de dieciocho años de edad ( $n=115047)$, el análisis incluyó 1086778 casos, de los cuales 25091 fueron por ERC. 


\section{CARACTERÍSTICAS DE LA MORTALIDAD POR ERC}

Incluyendo todos los años de observación, y en relación a todas las defunciones analizadas, la proporción de fallecimientos por ERC fue 2,3\% (IC 95\%: 2,28-2,34), y la proporción estandarizada fue 2,0 \% (IC 95\%: 1,95-2,04). La proporción estandarizada desagregada por región y año se describe en la Tabla 1.

La proporción de fallecimientos ajustada mostró mayor mortalidad en mujeres: 2,2 \% (IC 95\%: 2,2-2,3) versus 1,8 \% (IC 95\%: 1,8-1,9). La Figura 1 muestra la proporción estandarizada en relación a todas las muertes, indicando que la proporción de fallecimientos por ERC fue mayor en mujeres a través del periodo de observación. Dentro de los fallecimientos por ERC, la edad promedio aumentó de 70,3 años (DE: 17,2) en el 2003 a 72,9 años (DE: 15,9) en el 2015 ( $p$ de tendencia $<0,001$ ).

\section{TENDENCIA DE MORTALIDAD POR ERC}

La Figura 2 muestra los resultados del modelo de regresión lineal, es decir la mortalidad (proporción, \%) por ERC estimada a través de los años en cada región. Ninguna de las tendencias con pendiente negativa fue significativa; mientras que aquellas con pendientes positivas y diferentes de cero fueron (orden ascendente) en: Tacna $(\beta=0,91$, $p=0,006)$, La Libertad $(\beta=1,02, p=0,015)$, Tumbes $(\beta=1,04$, $p=0,001)$ Apurímac $(\beta=1,15, p=0,005)$, Cusco $(\beta=1,16$, $p=0,001)$, Ica $(\beta=1,25, \quad p=1,25)$, Moquegua $(\beta=1,42$,

Tabla 1. Números absolutos y proporción de muertes por enfermedad renal crónica (CIE 10: N18) por cada región y año, ajustado por edad y sexo

\begin{tabular}{|c|c|c|c|c|c|c|c|c|c|c|c|c|c|c|c|}
\hline \multirow[t]{2}{*}{ Región } & \multicolumn{2}{|c|}{$\begin{array}{l}\text { Todos los } \\
\text { años }\end{array}$} & \multirow{2}{*}{$\frac{2003}{\%}$} & \multirow{2}{*}{$\begin{array}{c}2004 \\
\%\end{array}$} & \multirow{2}{*}{$\begin{array}{c}2005 \\
\%\end{array}$} & \multirow{2}{*}{$\begin{array}{c}2006 \\
\%\end{array}$} & \multirow{2}{*}{$\frac{2007}{\%}$} & \multirow{2}{*}{$\begin{array}{c}2008 \\
\%\end{array}$} & \multirow{2}{*}{$\begin{array}{c}2009 \\
\%\end{array}$} & \multirow{2}{*}{$\begin{array}{c}2010 \\
\%\end{array}$} & \multirow{2}{*}{$\begin{array}{c}2011 \\
\%\end{array}$} & \multirow{2}{*}{$\frac{2012}{\%}$} & \multirow{2}{*}{$\begin{array}{c}2013 \\
\%\end{array}$} & \multirow{2}{*}{$\frac{2014}{\%}$} & \multirow{2}{*}{$\begin{array}{c}2015 \\
\%\end{array}$} \\
\hline & Número & $\%$ & & & & & & & & & & & & & \\
\hline Amazonas & 108 & 1,1 & 2,0 & 0,4 & 0,4 & 1,5 & 1,7 & 0,5 & 1,5 & 0,8 & 0,8 & 0,9 & 1,4 & 1,8 & 0,6 \\
\hline Ancash & 645 & 1,6 & 1,7 & 0,2 & 0,7 & 0,6 & 2,8 & 1,5 & 1,5 & 1,6 & 1,5 & 3,0 & 3,2 & 1,7 & 1,3 \\
\hline Apurímac & 461 & 1,8 & 0,9 & 1,4 & 1,5 & 2,2 & 1,2 & 0,9 & 1,6 & 2,5 & 1,8 & 2,4 & 2,1 & 3,0 & 1,5 \\
\hline Arequipa & 822 & 1,3 & 1,8 & 1,8 & 1,4 & 1,9 & 1,7 & 1,0 & 1,3 & 1,1 & 0,9 & 1,2 & 0,9 & 0,8 & 1,4 \\
\hline Ayacucho & 610 & 2,2 & 1,3 & 1,5 & 2,1 & 1,1 & 2,4 & 2,6 & 3,0 & 2,7 & 1,9 & 1,9 & 3,3 & 0,9 & 2,8 \\
\hline Cajamarca & 532 & 1,5 & 2,0 & 0,9 & 0,9 & 1,9 & 2,1 & 1,3 & 1,6 & 1,9 & 1,2 & 0,9 & 1,4 & 1,3 & 1,8 \\
\hline Callao & 1005 & 2,3 & 1,2 & 1,5 & 3,4 & 2,4 & 3,0 & 3,0 & 3,0 & 3,5 & 2,5 & 2,5 & 1,3 & 1,4 & 0,8 \\
\hline Cusco & 1280 & 2,0 & 1,6 & 2,2 & 1,5 & 2,0 & 2,2 & 2,8 & 3,3 & 1,4 & 1,7 & 1,7 & 1,9 & 0,9 & 3,1 \\
\hline Huancavelica & 563 & 2,2 & 2,4 & 1,5 & 3,0 & 2,1 & 2,5 & 2,3 & 4,1 & 1,6 & 2,8 & 1,6 & 0,5 & 1,5 & 1,3 \\
\hline Huánuco & 589 & 1,7 & 1,7 & 1,4 & 1,4 & 2,0 & 1,9 & 1,6 & 1,4 & 1,8 & 2,3 & 1,9 & 2,0 & 1,6 & 1,5 \\
\hline Ica & 1043 & 2,6 & 2,1 & 1,7 & 2,2 & 2,3 & 2,5 & 2,4 & 2,8 & 2,4 & 2,3 & 3,0 & 3,2 & 2,5 & 3,7 \\
\hline Junín & 1100 & 1,6 & 1,8 & 0,9 & 0,6 & 2,0 & 1,3 & 1,3 & 1,3 & 1,4 & 1,9 & 2,2 & 1,2 & 2,6 & 2,0 \\
\hline La Libertad & 1956 & 2,6 & 1,8 & 1,8 & 3,1 & 2,5 & 3,0 & 2,7 & 2,2 & 2,7 & 3,4 & 2,6 & 2,7 & 2,8 & 2,7 \\
\hline Lambayeque & 1277 & 2,4 & 1,8 & 2,1 & 2,6 & 3,0 & 2,8 & 1,6 & 2,2 & 2,6 & 2,3 & 2,7 & 2,4 & 2,7 & 2,6 \\
\hline Lima & 6138 & 1,7 & 1,3 & 1,0 & 2,3 & 2,4 & 2,7 & 1,6 & 1,8 & 1,5 & 1,4 & 1,4 & 1,4 & 1,3 & 1,8 \\
\hline Loreto & 192 & 1,8 & 0,5 & 1,0 & 2,4 & 1,8 & 2,1 & 2,3 & 1,6 & 1,4 & 2,1 & 1,8 & 2,6 & 1,3 & 2,5 \\
\hline Madre de Dios & 94 & 2,3 & 1,5 & 1,8 & 2,1 & 4,2 & 2,9 & 0,6 & 2,4 & 1,3 & 3,4 & 4,1 & 1,4 & 1,0 & 2,7 \\
\hline Moquegua & 217 & 2,7 & 2,0 & 1,7 & 2,7 & 1,9 & 2,5 & 2,6 & 3,9 & 2,7 & 1,4 & 6,5 & 1,3 & 3,5 & 5,6 \\
\hline Pasco & 173 & 1,7 & 1,5 & 2,1 & 2,8 & 1,1 & 1,0 & 0,9 & 1,3 & 1,1 & 3,5 & 2,6 & 2,4 & 2,3 & 1,6 \\
\hline Piura & 1210 & 1,7 & 1,7 & 1,2 & 1,6 & 1,5 & 1,4 & 1,2 & 0,9 & 1,1 & 1,8 & 2,1 & 2,1 & 2,7 & 2,4 \\
\hline Puno & 4055 & 4,1 & 4,0 & 3,4 & 3,4 & 3,5 & 4,8 & 5,3 & 5,3 & 3,7 & 3,3 & 3,7 & 4,1 & 4,0 & 4,2 \\
\hline San Martin & 283 & 2,0 & 1,1 & 1,0 & 1,6 & 4,1 & 2,5 & 2,5 & 1,8 & 1,8 & 1,6 & 1,8 & 0,6 & 2,2 & 3,2 \\
\hline Tacna & 321 & 2,2 & 2,8 & 2,2 & 3,5 & 2,7 & 3,3 & 2,7 & 2,4 & 1,5 & 1,3 & 0,9 & 2,4 & 2,2 & 1,6 \\
\hline Tumbes & 167 & 2,5 & 1,9 & 2,6 & 2,1 & 0,4 & 1,8 & 2,4 & 0,8 & 1,9 & 3,6 & 0,8 & 2,9 & 3,8 & 9,5 \\
\hline Ucayali & 250 & 1,9 & 1,7 & 1,0 & 1,1 & 2,9 & 2,4 & 0,4 & 1,5 & 1,9 & 3,4 & 2,2 & 2,2 & 2,8 & 2,1 \\
\hline Total & 25091 & 2,0 & 1,7 & 1,4 & 2,1 & 2,2 & 2,5 & 2,0 & 2,1 & 1,9 & 1,9 & 2,0 & 1,9 & 1,9 & 2,2 \\
\hline
\end{tabular}

Los porcentajes han sido estandarizados por sexo y edad; los porcentajes indican la proporción de muertes por enfermedad renal crónica en relación a todos los fallecimientos en la región y año. Interpretación (ejemplo): en todos los años de observación, la proporción de muertes por enfermedad renal crónica fue $1,9 \%$ en Ucayali 


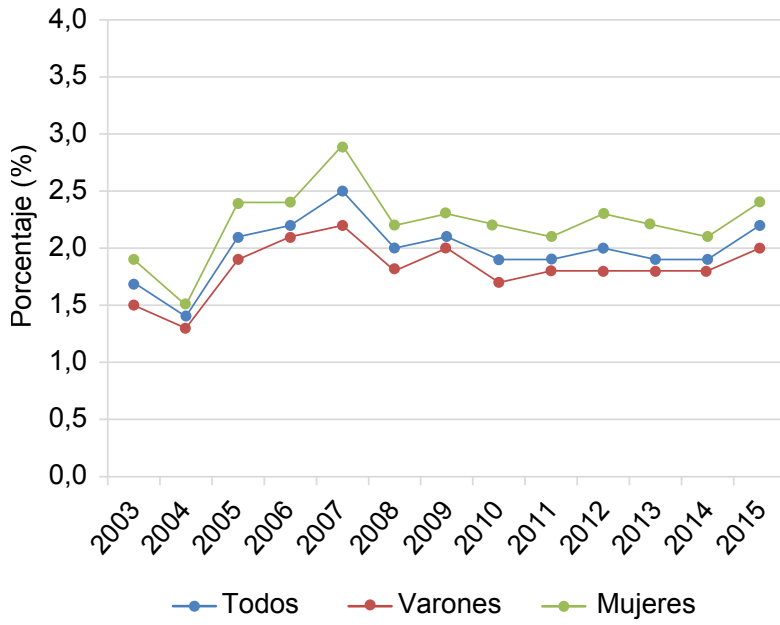

Las proporciones presentadas están ajustadas por edad y sexo.

Figura 1. Proporción de muertes por enfermedad renal crónica (CIE-10: N18) para toda la población según sexo a través de los años de estudio

$p=0,001)$, Ayacucho $(\beta=1,45, p=0,001)$, Huancavelica $(\beta=2,01, p<0,001)$, y Puno $(\beta=4,52, p<0,001)$. Esto significa que, en comparación a las demás regiones, la de interés empezó el periodo de observación con una proporción de muertes por ERC $\beta$ veces mayor. Por ejemplo, en comparación con las demás regiones, Puno empezó el periodo de observación con $4,5 \%$ más muertes por ERC. Por otro lado, la variable del año en el modelo de regresión tuvo una pendiente positiva $(\beta=0,06, p=0,001)$, indicando que, dado el punto de inicio de cada región, la proporción de muertes por ERC aumentó en 0,06 puntos porcentuales cada año.

\section{DISCUSIÓN}

La proporción de muertes por ERC en relación al total de los fallecimientos en cada año, ha aumentado desde el 2003. El aumento en los casos de muerte por ERC es sostenido en la mayoría de regiones del país, con algunas excepciones (4 de 25 regiones). Es necesario destinar recursos para prevenir, identificar precozmente, o iniciar tratamiento para ERC en varias -o todas- regiones del país para disminuir el impacto de esta patología en la población.

La edad de muerte por ERC aumentó progresivamente. Esto va de acuerdo con la mayor esperanza de vida, o puede explicarse por más acceso a tratamiento, el mismo que puede retrasar el desenlace fatal. La mortalidad fue mayor en mujeres; este hallazgo discrepa con lo descrito en pacientes en hemodiálisis ${ }^{(12)}$, pero concuerda a lo descrito cuando se analizan otras terapias de reemplazo renal ${ }^{(13)}$. La diferencia en género podría explicarse por el cuidado de la salud. El varón suele buscar atención médica cuando hay síntomas severos o limitantes, es decir enfermedad avanzada que puede presentar otras complicaciones y así causas de muerte. Un estudio en un hospital local reportó una tendencia a menor mortalidad en pacientes con ERC en diálisis si el diagnóstico había sido hecho más de un mes atrás y si el tratamiento había sido planeado (no emergencia) ${ }^{(12) .}$.

Lima tuvo una relativa baja tasa de muertes por ERC, quizá porque es en dónde se concentra la mayor cantidad de especialistas (p. ej. nefrólogos) ${ }^{(10,14)}$ y de centros con capacidad de tratamiento para ERC. A pesar de estos beneficios, la proporción de muertes puede ser por la excesiva demanda, porque pacientes de otras ciudades migran en estadios avanzados buscando mejor atención, o por otras características epidemiológicas (p. ej. frecuencia más alta de otros factores de riesgo); además, estos resultados para Lima pueden reflejar la gran población que reside en esta ciudad (aproximadamente un tercio de la población nacional). En cualquier caso, los servicios de prevención pueden mejorar, con énfasis en poblaciones de riesgo como pacientes con hipertensión o diabetes ${ }^{(15)}$. Esto puede ser particularmente relevante en Lima, ciudad que exhibe más prevalencia por estas dolencias crónicas, a las cuales se le puede atribuir el $44 \%$ (nefropatía diabética) y $24 \%$ de los casos de ERC ${ }^{(16)}$.

Las ciudades que mostraron aumento en la proporción de muertes por ERC son aquellas en donde no hay, o hay muy pocos nefrólogos ${ }^{(10)}$. La responsabilidad no sólo recae en esta especialidad, y la única solución no puede ser proveer de más nefrólogos. Otras especialidades pueden apoyar en la detección. Por ejemplo, a nivel local se recomienda que el médico general o de familia identifique poblaciones con factores de riesgo; diagnostique precozmente la ERC; participe en la referencia y contrareferencia con el especialista; entre otras medidas ${ }^{(17)}$. Estas estrategias, y otras complementarias, deberían ser seguidas por aquellos involucrados en el manejo de pacientes con diabetes o hipertensión.

Pacientes con enfermedades predisponentes tampoco deben ser los únicos en quienes se descarte la ERC, lo cual se puede hacer con pruebas de laboratorio o evaluación clínica con cuestionarios validados ${ }^{(18)}$. Recientemente se ha propuesto uno para el Perú, el cual tiene una aceptable capacidad para descartar casos prevalentes ( $p$. ej. alto valor predictivo negativo) ${ }^{(19)}$. Si bien es aún prematuro recomendar su aplicación, es una herramienta disponible que debe ser estudiada para fomentar su utilización. Las herramientas de tamizaje pueden ayudar a reducir los costos de diagnóstico, identificando a aquellos que se beneficiarían más de exámenes de laboratorio para acercarse al diagnóstico final.

Puno ( 3,800 msnm) presentó altas tasas de mortalidad por ERC y continúa en ascenso; si bien, en menor magnitud, Huancavelica $(\sim 3,600 \mathrm{msnm})$ también presentó una tendencia con pendiente positiva. Se ha descrito que 

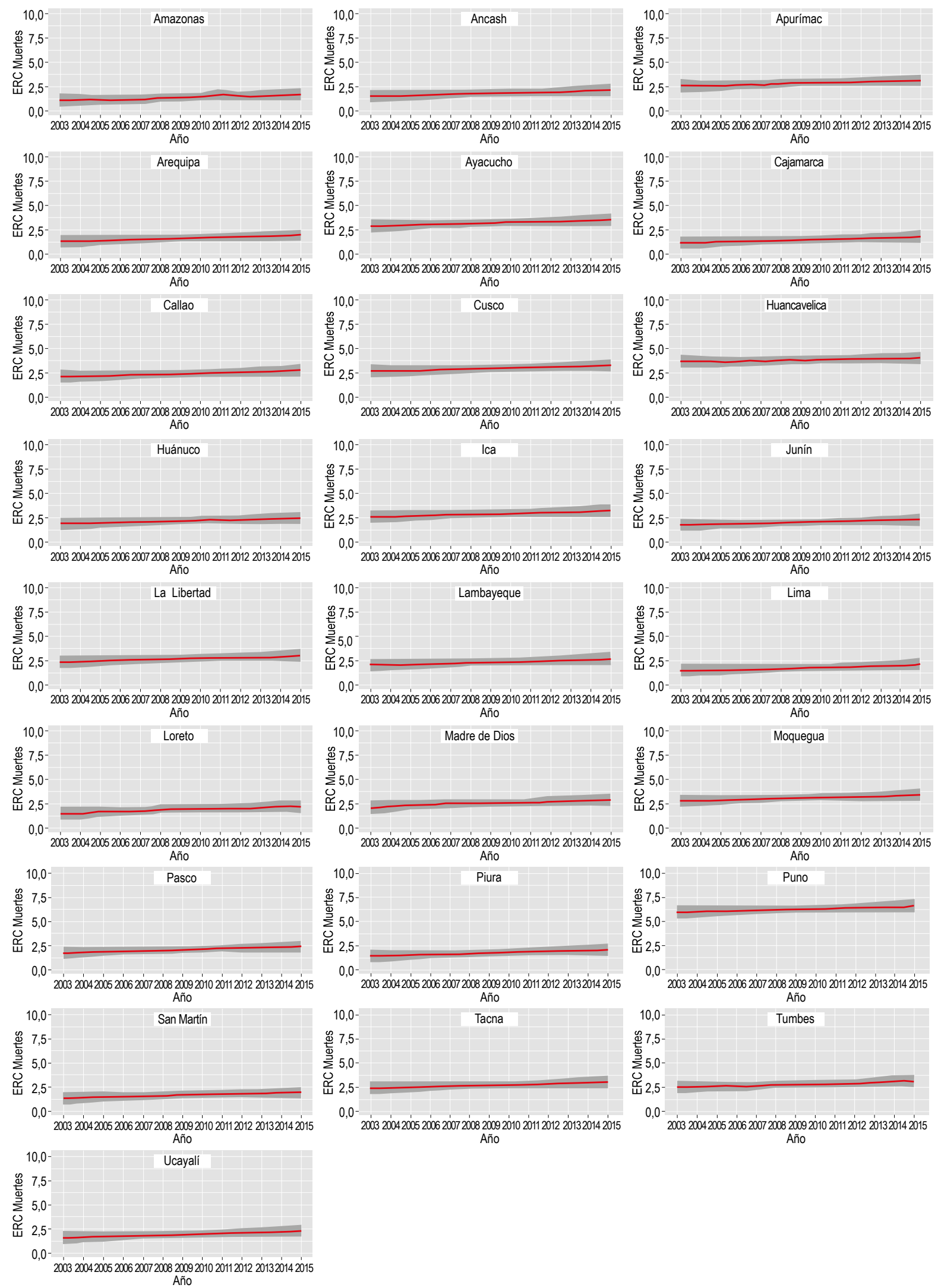

ERC: enfermedad renal crónica

Figura 2. Proporción de mortalidad (expresada en porcentaje) por enfermedad renal crónica (CIE 10: N18) a través de los años en en cada región del Perú 
ciudades de altura en el Perú, en comparación a otras al nivel del mar, tienen mayor riesgo de desarrollar diabetes mellitus del tipo $2{ }^{(5)}$. Esto podría explicar el aumento en la mortalidad, pues más casos de diabetes están apareciendo sin adecuado control metabólico ni de otras comorbilidades. La altura podría jugar también un rol relevante ${ }^{(20)}$, como en el caso del síndrome renal de altura ${ }^{(21)}$. En cualquier caso, sea por enfermedades no transmisibles o exposición crónica a la altura, este hallazgo merece mayor atención e investigación para mejorar la situación.

La caída en la proporción de defunciones por ERC después del 2007 se puede deber a que, a partir de este año, el MINSA empezó a financiar completamente el acceso a la terapia de reemplazo renal ${ }^{(10)}$. De esta manera, más personas con ERC pudieron acceder al tratamiento. El incremento descrito en los años posteriores puede ser por no continuar con este programa de manera óptima, o porque los casos de ERC se diagnostican en etapas más avanzadas, lo que va de acuerdo a los niveles más altos -prevalencia e incidencia- de otros factores de riesgo ${ }^{(5,7)}$.

Si fuera posible descartar la presencia de factores de riesgo conocidos para ERC (p. ej. diabetes mellitus tipo 2 e hipertensión arterial), se podría hablar de ERC de etiología desconocida. Para dicha condición se han descrito otros factores de riesgo como deshidratación crónica (frecuente en sujetos que trabajan en el campo) ${ }^{(22)}$, exposición a pesticidas (aunque la evidencia es limitada) ${ }^{(23,24)}$, e infección por leptospirosis (evidencia aún escasa) ${ }^{(25)}$. Estas causas, aunque secundarias en relación a los factores de riesgo preponderantes, se deben tener en mente para no sólo sospechar -y así descartar- ERC en pacientes con antecedentes de diabetes o hipertensión.

Este estudio se beneficia del uso de registros de mortalidad de alcance nacional, los mismos que están disponibles para un periodo de más de 10 años. Los registros utilizados se basan en certificados de defunción que, si bien pueden tener limitaciones, ofrecen información posiblemente más robusta en comparación a aquella recolectada por cuestionarios u otros métodos de entrevista sin procesos de adjudicación de eventos. Entre las limitaciones del estudio destaca la falta de variables sociodemográficas (p. ej. grado de educación, nivel socioeconómico u ocupación laboral) para describir a los occisos; tampoco fue posible acceder a variables clínicas (p. ej. si recibe diálisis, estadio de ERC o edad de diagnóstico) para describir el estado general o patológico del fenecido, así como su impacto en la mortalidad.

Dado que los datos provienen de los registros compilados por el MINSA, existe potencial subregistro de establecimientos de salud fuera de este sistema (p. ej. establecimientos privados) o aquellos sucedidos fuera de establecimientos de salud. Por lo tanto, nuestros resultados subestiman la cantidad de defunciones, alertando de una posible mayor magnitud del problema. De igual manera, no se calculó la tasa de mortalidad por no tener el denominador exacto; es decir, población en riesgo en cada año. El presente estudio buscó describir la mortalidad por ERC, describir la causa exacta de la muerte ( $p$. ej. causa intermedia o final) en pacientes cuya patología de base (p. ej. causa subyacente) fue la ERC, escapa al objetivo del presente estudio y debería ser tema de análisis posteriores para expandir y confirmar otras observaciones locales ${ }^{(26)}$.

Entre 2003 y 2015, la mortalidad por enfermedad renal crónica en el Perú ha aumentado, y la misma tendencia se observa en varias regiones del país. Es necesario implementar medidas de prevención, temprana identificación, y acceso al tratamiento, para detener esta tendencia.

Contribución de los autores: Ambos autores concibieron la idea de investigación. RMCL desarrolló el análisis estadístico con la colaboración de $\mathrm{ABO}$. RMCL escribió el primer borrador del artículo. $A B O$ editó críticamente el artículo. Ambos autores aceptaron el contenido final del artículo.

Fuentes de financiamiento: $A B O$ recibe financiamiento como Research Training Fellow in Public Health and Tropical Medicine (103994/Z/14/Z) del Wellcome Trust.

\section{Conflicto de Intereses: Ninguno}

\section{REFERENCIAS BIBLIOGRÁFICAS}

1. Global, regional, and national age-sex specific mortality for 264 causes of death, 1980-2016: a systematic analysis for the Global Burden of Disease Study 2016. Lancet 2017;390(10100):1151-210.

2. Global, regional, and national disabilityadjusted life-years (DALYs) for 333 diseases and injuries and healthy life expectancy (HALE) for 195 countries and territories, 1990-2016: a systematic analysis for the Global Burden of Disease Study 2016. Lancet 2017;390(10100):1260-344.
3. Herrera-Añazco P, Pacheco-Mendoza J, Taype-Rondan A. La enfermedad renal crónica en el Perú. Una revisión narrativa de los artículos científicos publicados. Acta Med Peru. 2016;33(2):130-7.

4. Francis ER, Kuo CC, Bernabe-Ortiz A, Nessel L, Gilman RH, Checkley W, et al. Burden of chronic kidney disease in resource-limited settings from Peru: a population-based study. BMC Nephrol 2015;16:114.

5. Bernabe-Ortiz A, Carrillo-Larco RM, Gilman RH, Miele CH, Checkley W,
Wells JC, et al. Geographical variation in the progression of type 2 diabetes in Peru: The CRONICAS Cohort Study. Diabetes Res Clin Pract 2016;121:135-45.

6. Bernabe-Ortiz A, Sanchez JF, CarrilloLarco RM, Gilman RH, Poterico JA, Quispe R, et al. Rural-to-urban migration and risk of hypertension: longitudinal results of the PERU MIGRANT study. J Hum Hypertens 2017;31(1):22-8.

7. Bernabe-Ortiz A, Carrillo-Larco RM, Gilman RH, Checkley W, Smeeth L, 
Miranda JJ. Impact of urbanisation and altitude on the incidence of, and risk factors for, hypertension. Heart 2017;103(11):827-33.

8. Seclen SN, Rosas ME, Arias AJ, Medina CA. Elevated incidence rates of diabetes in Peru: report from PERUDIAB, a national urban population-based longitudinal study. BMJ Open Diabetes Res Care 2017;5(1):e000401.

9. Seclen SN, Rosas ME, Arias AJ, Huayta E, Medina CA. Prevalence of diabetes and impaired fasting glucose in Peru: report from PERUDIAB, a national urban population-based longitudinal study. BMJ Open Diabetes Res Care 2015;3(1):e000110.

10. Ministerio de Salud del Perú. Análisis de la situación de la enfermedad renal crónica en el Perú, 2015 [Internet]. Lima: Dirección General de Epidemiologia, MINSA; 2015. Disponible en: http://www.dge.gob.pe/portal/index. php?option $=$ com_content $\&$ view $=$ article\&id=598\&Itemid $=353$

11. The R Project for Statistical Computing [Internet]. Vienna: The R Foundation; 2017. Disponible en: https://www.Rproject.org

12. Herrera-Anazco P, Benites-Zapata V, Hernandez AV, Mezones-Holguin E, Silveira-Chau M. Mortality in patients with chronic kidney disease undergoing hemodialysis in a public hospital of Peru. J Bras Nefrol 2015;37(2):192-7.

13. Cieza Zevallos J, Jeanette BH, Zegarra Montes L, Ortiz Soriano V, León Rabanal C. Supervivencia en terapias de reemplazo renal dentro de un concepto integral de oferta de servicios públicos en el Perú, periodo 2008 y 2012. Acta Méd Peru. 2013;30(4):80-5.

14. Zevallos L, Pastor R, Moscoso B. Oferta y demanda de médicos especialistas en los establecimientos de salud del Ministerio de Salud: brechas a nivel nacional, por regiones y tipo de especialidad. Rev Peru Med Exp Salud Publica 2011;28(2):177-85.

15. Chapter 1: Definition and classification of CKD. Kidney Int Suppl (2011). 2013;3(1):19-62.

16. Salman $M$, Khan AH, Adnan AS, Sulaiman SA, Hussain K, Shehzadi N, et al. Attributable causes of chronic kidney disease in adults: a five-year retrospective study in a tertiary-care hospital in the northeast of the Malaysian Peninsula. Sao Paulo Med J 2015;133(6):502-9.

17. Palacios Guillén AM, Medina Santander B, Campos Buleje SC, Berríos Medina EC, Solís Vásquez G, Bravo Zúñiga JI, et al. Guía Clínica para Identificación, Evaluación y Manejo Inicial del Paciente con Enfermedad Renal Crónica en el Primer Nivel de Atención [Internet]. Lima: Sociedad Peruana de Nefrología; 2010. Disponible en: http://www.spn.pe/archivos/guias_spn/ PARA_IDENTIFICACION_EVALUACION_Y_MANEJO_INICIAL_ DEL_PACEINTE_CON_ERC_EN_ EL_PRIMER_NIVEL_DE_ATENCION.pdf

18. Echouffo-Tcheugui JB, Kengne AP. Risk models to predict chronic kidney disease and its progression: a systematic review. PLoS Med 2012;9(11):e1001344.

19. Carrillo-Larco RM, Miranda JJ, Gilman RH, Medina-Lezama J, Chirinos-Pacheco JA, Munoz-Retamozo PV, et al. Risk score for first-screening of prevalent undiagnosed chronic kidney disease in Peru: the CRONICAS-CKD risk score. BMC Nephrol 2017;18(1):343.

20. Hurtado-Arestegui A, Plata-Cornejo R, Cornejo A, Mas G, Carbajal L, Sharma S, et al. Higher prevalence of unrecognized kidney disease at high altitude. J Nephrol 2018;31(2):263-9.
21. Hurtado A, Escudero E, Pando J, Sharma S, Johnson RJ. Cardiovascular and renal effects of chronic exposure to high altitude. Nephrol Dial Transplant 2012;27 Suppl 4:iv11-6.

22. Wegman DH, Apelqvist J, Bottai M, Ekstrom U, Garcia-Trabanino R, Glaser J, et al. Intervention to diminish dehydration and kidney damage among sugarcane workers. Scand J Work Environ Health 2018;44(1):16-24.

23. Valcke M, Levasseur ME, Soares da Silva A, Wesseling C. Pesticide exposures and chronic kidney disease of unknown etiology: an epidemiologic review. Environ Health 2017;16(1):49.

24. Ghosh R, Siddarth M, Singh N, Tyagi V, Kare PK, Banerjee BD, et al. Organochlorine pesticide level in patients with chronic kidney disease of unknown etiology and its association with renal function. Environ Health Prev Med 2017;22(1):49.

25. Yang CW. Leptospirosis Renal Disease: Emerging Culprit of Chronic Kidney Disease Unknown Etiology. Nephron 2018;138(2):129-36.

26. Concepción-Zavaleta M, CorteganaAranda J, Ocampo-Rujel N, GutiérrezPortilla W. Factores de riesgo asociados a mortalidad en pacientes con enfermedad renal crónica terminal. Rev Soc Peru Med Interna 2015;28(2):72-8.

Correspondencia: Antonio Bernabé-Ortiz, $M D, M P H, P h D$

Dirección: CRONICAS Centro de Excelencia en Enfermedades Crónicas, Universidad Peruana Cayetano Heredia, Av. Armendáriz 497, Lima 18, Perú

Teléfono: 511-2416978

Correo electrónico: Antonio.Bernabe@upch.pe 